\title{
LA NUEVA LEY DE SERVICIOS SOCIALES DE ANDALUCÍA: UNA PRESENTACIÓN
}

\author{
María Antonia CAStro ArgüElles \\ Catedrática de Derecho del Trabajo y de la Seguridad Social \\ Facultad de Derecho de la Universidad de Oviedo \\ mcastro@uniovi.es
}

\section{LA RENOVACIÓN DE LA LEGISLACIÓN ANDALUZA DE SERVICIOS SOCIALES}

El 21 de enero de 2017 se publicaba en el BOE la Ley 9/2016, de 27 de diciembre, de Servicios Sociales de Andalucía, que había entrado en vigor pocos días antes, el 18 de enero ${ }^{1}$. Esta Ley viene a sustituir a la Ley 2/1988, de 4 de abril, a la que deroga expresamente (disposición derogatoria primera), que en su momento se había ocupado de configurar en la Comunidad Autónoma de Andalucía «un Sistema Público de Servicios Sociales».

De esta forma Andalucía se suma a la tendencia seguida por la mayoría de las Comunidades Autónomas de aprobación de una «segunda generación» de leyes autonómicas de servicios sociales ${ }^{2}$ que ofrecen una regulación mucho más extensa y con un mayor nivel de desarrollo ${ }^{3}$. Ésta es, desde luego, la impresión que produce una primera lectura de la Ley 9/2016, aunque solo sea porque frente a los 33 artículos repartidos en seis títulos, seis disposiciones adicionales, dos disposiciones transitorias, una derogatoria y dos finales de la Ley 2/1988, el nuevo texto normativo consta de 141 artículos organizados en el mismo número de títulos, a los que, además, se suma uno preliminar, el mismo número de disposiciones adicionales y una disposición transitoria y otra final más de las que se incluían en la Ley 2/1988.

\footnotetext{
${ }^{1}$ Según su disposición final tercera: «La presente ley entrará en vigor a los veinte días de su publicación en el Boletín Oficial de la Junta de Andalucía», lo que tuvo lugar el 29 de diciembre de 2016.

2 En la mayoría de Comunidades Autónomas las revisiones normativas en la materia corresponden al periodo 2003-2010. Sobre el tema vid. V. AGUADO I CudOLÀ, «El régimen jurídico de las prestaciones de los servicios sociales», en A. EzQuerRA Huerva (coord.), El marco jurídico de los servicios sociales en España, Barcelona, Atelier, 2012, p. 51.

3 En este sentido vid. J. M. Pemán Gavín, «Prólogo», en A. Ezquerra Huerva (coord.), El marco jurídico de los servicios sociales en España, Barcelona, Atelier, 2012, p. 16.
} 
Esta renovación de la legislación autonómica en el ámbito de los servicios sociales se debe, en gran medida, a las reformas de los Estatutos de Autonomía, que han incorporado referencias expresas a unos derechos «íntimamente relacionados con las políticas sociales» (según la exposición de motivos de la Ley 9/2016) para avanzar, supuestamente, en el papel que los poderes públicos autonómicos pueden tener, dentro de su ámbito de competencias, en el desarrollo de los encargos contenidos en la Constitución ${ }^{4}$. Entre esos derechos figuran los derechos fundamentales de la Constitución, pero también, formulados como derechos, los principios rectores de la política social y económica (recogidos en los arts. 39, 48,49 y $50 \mathrm{CE}$ ) que sirven de apoyo para orientar la actuación de protección social a determinados grupos de población (la familia y, en especial, los hijos; la juventud; las personas con discapacidad física, sensorial y psíquica, y las personas mayores).

En el concreto caso de Andalucía, la reforma de su Estatuto de Autonomía se llevó a cabo por la Ley Orgánica 2/2007, de 19 de marzo, que dedica un título a los derechos sociales (título I) en el que se incluye una mención expresa al «derecho de todos a acceder en condiciones de igualdad a las prestaciones de un Sistema Público de Servicios Sociales» (art. 23.1). Junto a este derecho se formulan los derechos a la igualdad de género (art. 15), a la protección contra la violencia de género (art. 16), a la protección de la familia (art. 17), de personas menores (art. 18), de personas mayores (art. 19), de personas con discapacidad o dependencia (art. 24), al acceso de todas las personas en condiciones de igualdad a las prestaciones de un Sistema Público de Servicios Sociales (art. 23.1) y a una renta básica que garantice unas condiciones de vida dignas (art. 23.2).

En desarrollo de estas previsiones, la Ley 9/2016 se propone promover y garantizar en el ámbito de la Comunidad Autónoma «el derecho universal» de todas las personas a las prestaciones y servicios del Sistema Público de Servicios Sociales. Más en concreto, como se verá, la Ley 9/2016 ordena y regula el acceso a un grupo de prestaciones (las prestaciones garantizadas) como derecho subjetivo, dentro del marco de los requisitos de acceso que se regulen en cada caso; algo que ya se había hecho antes, en el ámbito estatal, en una parcela muy concreta de la protección social como es la dependencia, con la Ley 39/2006, de 14 de diciembre.

\footnotetext{
${ }^{4}$ A favor vid. V. Aguado I Cudolà, «El régimen jurídico...»», op. cit., p. 51, quien, no obstante, hace referencia a sectores doctrinales críticos que han visto en ello «la deconstrucción política del Estado social y democrático de Derecho, y sobre todo la ruptura de la unidad de la nación española».
} 
Con todo, actualizar el régimen jurídico del sistema de servicios sociales creado por la Ley 2/1988 de acuerdo con la nueva redacción del Estatuto de Autonomía no ha sido la única razón apuntada por la Ley 9/2016 para justificar su aprobación. La exposición de motivos de dicha Ley alude también al propósito de adaptarse mejor a la evolución de la sociedad y atender a las exigencias que imponen la aparición de nuevas necesidades sociales. En concreto, el objetivo declarado de la nueva Ley ha sido «consolidar, fortalecer y mejorar» la capacidad de adaptación del sistema de servicios sociales ya existente para asegurar así una protección integral a la ciudadanía.

Para la nueva Ley el Sistema Público de Servicios Sociales de Andalucía sigue siendo el conjunto de servicios, recursos y prestaciones orientados a garantizar el derecho de todas las personas en Andalucía a la protección social, la promoción social y la prevención, en los términos recogidos en el Estatuto de Autonomía y en la normativa vigente en la materia (art. 24). Su finalidad de «favorecer la integración social, la igualdad de oportunidades, la autonomía personal, la convivencia y la participación social, y el bienestar social de todas las personas, familias y grupos, desarrollando una función promotora, preventiva, protectora y asistencial» (art. 2.5) no difiere sustancialmente de la prevista en Ley 2/1988, que hablaba de promocionar el desarrollo de los individuos y los grupos para la obtención de mayor bienestar social, de la prevención de situaciones de riesgo y de la compensación de déficits de apoyo social.

La Ley 9/2016 deja al desarrollo reglamentario algunos aspectos regulatorios esenciales para su implantación y desarrollo, como el Catálogo de Prestaciones del Sistema Público de Servicios Sociales, en particular, la definición de cada uno de los servicios y prestaciones ofrecidas, su ámbito y alcance, las condiciones requeridas para acceder a los mismos y su disponibilidad dentro del sistema, o el Plan Estratégico de Servicios Sociales, al que identifica como uno de los «elementos fundamentales y dinamizadores del desarrollo de los objetivos de la política social en Andalucía». Ese plan deberá programar, con la periodicidad que se determine en el mismo, las prestaciones, servicios, programas y otras actuaciones necesarias para cumplir los objetivos del sistema de servicios sociales de Andalucía.

Entre tanto se proceda al desarrollo reglamentario de esta Ley, seguirán siendo de aplicación las normas actualmente vigentes, dictadas en desarrollo de la Ley 2/1988, en lo que no sean contrarias, y en tanto no sean sustituidas ni derogadas (disposición derogatoria segunda). Entre esas normas están, además de las encargadas de regular el reconocimiento y disfrute de 
las distintas prestaciones, las que se han dictado desde la puesta en funcionamiento del sistema en desarrollo de las previsiones contenidas en la Ley, «con el fin de atender a distintos sectores o grupos poblacionales», de acuerdo con sus necesidades y especificidades 5 .

\section{LAS COMPETENCIAS DE LA COMUNIDAD AUTÓNOMA DE ANDALUCÍA EN MATERIA DE SERVICIOS SOCIALES}

El Estatuto de Autonomía de Andalucía establece las competencias exclusivas de la Comunidad Autónoma en materia de servicios sociales. Así se recogía en el art. 13.22 de la Ley Orgánica 6/1981, de 30 de diciembre, y se reitera en el art. 61 de la Ley Orgánica 2/2007, de 19 de marzo, de Reforma del Estatuto de Autonomía para Andalucía. Una opción que se ha apoyado en todo momento en el art. 148.1.20 CE según el cual las Comunidades Autónomas podrán asumir competencias en materia de asistencia social; ámbito material en el que se han venido incluyendo los servicios sociales.

A esa identificación de los servicios sociales con la asistencia social ha contribuido, sin duda, el concepto amplio de servicios sociales utilizado por el legislador autonómico ${ }^{6}$, que incorpora prestaciones tanto técnicas o de servicios como económicas, propias de la asistencia social ${ }^{7}$, sin que haya supuesto ningún problema que esos servicios sociales incluyan

5 Por cierto, que la relación de normas que se menciona de forma expresa en la exposición de motivos de la Ley 9/2016 permite hacerse cargo de a qué grupos de población y a qué necesidades se refiere el legislador autonómico: la Ley 4/1997, de 9 de julio, de Prevención y Asistencia en Materia de Drogas; la Ley 1/1998, de 20 de abril, de los Derechos y la Atención al Menor; la Ley 1/1999, de 31 de marzo, de Atención a las Personas con Discapacidad de Andalucía; la Ley 6/1999, de 7 de julio, de Atención y Protección a las Personas Mayores; la Ley 5/2002, de 16 de diciembre, de Parejas de Hecho; la Ley 1/2009, de 27 de febrero, Reguladora de la Mediación Familiar en la Comunidad Autónoma de Andalucía; la Ley 12/2007, de 26 de noviembre, para la Promoción de la Igualdad de Género en Andalucía; la Ley 13/2007, de 26 de noviembre, de Medidas de Prevención y Protección Integral contra la Violencia de Género, y la Ley 11/2011, de 5 de diciembre, por la que se regula el uso de la lengua de signos española y los medios de apoyo a la comunicación oral de las personas sordas, con discapacidad auditiva y con sordoceguera en Andalucía.

${ }^{6}$ Tanto en la Ley 2/1988, de 4 de abril, al definir el Sistema Público de Servicios Sociales, como en el Estatuto de Autonomía al delimitar las competencias de la Comunidad Autónoma en materia de servicios sociales (art. 61 de la Ley Orgánica 2/2007).

${ }^{7}$ Sobre el tema vid. J. Tornos Mas y A. Galán Galán, «La configuración de los servicios sociales como servicio público. Derecho subjetivo de los ciudadanos a la prestación de servicios», Proyecto FIPROS/1051/2015, MTAS, p. 7, para quienes la asistencia social se identifica con las prestaciones dinerarias y los servicios sociales con las prestaciones técnicas o en servicios. 
también otro tipo de prestaciones o que no tengan por qué tener en todo caso carácter asistencial ni, por ello, vincularse necesariamente a estados o situaciones de necesidad.

Pero los servicios sociales también se encuentran materialmente relacionados con la Seguridad Social. En efecto, según el Texto Refundido de la Ley General de la Seguridad Social (aprobado por Real Decreto Legislativo 8/2015), forman parte de la acción protectora del sistema de Seguridad Social las prestaciones de servicios sociales que pueden establecerse en materia de formación y rehabilitación de personas con discapacidad y de asistencia a las personas mayores, así como en aquellas otras materias que se considere conveniente [art. 42.1.e) TRLGSS]. En concreto, el art. 63 TRLGSS incorpora una referencia a las prestaciones de servicios sociales que dispensará la Seguridad Social como complemento de las prestaciones correspondientes a las situaciones específicamente protegidas por el sistema de la Seguridad Social. Por cierto que, en este ámbito, esa alusión a los servicios sociales aparece conceptualmente separada de la referida a los beneficios de asistencia social a que alude el art. 42.2 TRLGSS, que podrán otorgarse como complemento de las prestaciones de la Seguridad Social ${ }^{8}$, precisamente porque los primeros no han de tener necesariamente carácter asistencial. En cualquier caso, al tratarse de prestaciones y beneficios integrados en la acción protectora del sistema de la Seguridad Social es claro que las competencias que puedan asumir las Comunidades Autónomas al respecto deberán apoyarse en el art. 149.1.17 CE y en las posibles competencias de ejecución de los servicios de la Seguridad Social, y no en el antes mencionado art. 148.1.20 CE.

Por tanto, los servicios sociales constituyen una técnica prestacional que puede ser incluida por la Seguridad Social o quedar fuera como protección social externa al sistema, por lo que las competencias en esta materia no pueden ser asumidas en exclusiva ni por el Estado ni por las Comunidades Autónomas9. Y así parecía haberlo entendido la Ley 2/1988, de 4 de abril, de Servicios Sociales de Andalucía, al proponerse como objetivo la configuración de un sistema público que unificase y ordenase el pano-

\footnotetext{
${ }^{8}$ En concreto, el art. 64 TRLGSS incluye en la noción de asistencia social del sistema de la Seguridad Social los «servicios y auxilios económicos» que se podrán dispensar a las personas incluidas en su campo de aplicación y a los familiares o asimilados que de ellas dependan, en atención a estados y situaciones de necesidad que se consideren precisos, previa demostración de que el interesado carece de los recursos indispensables para hacer frente a tales estados o situaciones de necesidad.

9 J. A. Fernández Avilés, «Artículo 53», en J. García Murcia (dir.), Ley General de Seguridad Social. Comentada y con jurisprudencia, Madrid, La Ley, 2009, p. 406.
} 
rama organizativo previo que había resultado del proceso de transferencia de competencias del Estado en materia de servicios sociales que tuvo lugar en los ochenta ${ }^{10}$. Ese proceso, como se recordará, culminó, precisamente, con el Real Decreto 1752/1984, de 1 de agosto, de traspaso a la Comunidad Autónoma de Andalucía de funciones y servicios del Instituto Nacional de Servicios Sociales (INSERSO) de la Seguridad Social, creado para la gestión de la asistencia social y servicios sociales complementarios de las prestaciones básicas del sistema. Por ello, en el articulado de la Ley 2/1988 había referencias a los servicios sociales de la Seguridad Social que, como no podía ser de otra manera, se limitaban a aspectos de gestión (art. 20). Esa gestión debía desarrollarse conforme a la normativa vigente de la Seguridad Social (disposición adicional segunda), de manera que, en este momento, el legislador autonómico, aunque se propusiera esa «unificación y ordenación» de todos los servicios sociales, era consciente de que las competencias respecto de los transferidos servicios sociales de la Seguridad Social quedaban limitadas por lo dispuesto en el art. 149.1.17 CE, aunque no se dijera expresamente.

Posteriormente, la nueva versión del Estatuto de Autonomía de Andalucía (LO 2/2007) ha reiterado la atribución a la Comunidad Autónoma de la competencia exclusiva en materia de servicios sociales, pero lo ha hecho de una forma más detallada. En el nuevo Estatuto de Autonomía esas competencias en materia de servicios sociales incluyen (art. 61.1): la regulación, ordenación y gestión de servicios sociales; las prestaciones técnicas y las prestaciones económicas con finalidad asistencial o complementarias de otros sistemas de protección pública, y la regulación y la aprobación de planes y programas específicos dirigidos a personas y colectivos en situación de necesidad social. En materia de Seguridad Social, el Estatuto se limita a reconocer a la Comunidad Autónoma las competencias ejecutivas (art. 63). Finalmente, según el art. 84, la Comunidad Autónoma podrá organizar y administrar todos los servicios relacionados con educación, sanidad y servicios sociales, y ejercerá la tutela de las instituciones y entidades en estas materias, sin perjuicio de la alta inspección del Estado.

La Ley 9/2016 asume, como no podía ser de otra manera, esas competencias en materia de servicios sociales, pero omite cualquier referen-

10 Téngase en cuenta que con el Real Decreto 251/1982, de 15 de marzo, se transfirieron las competencias, funciones y servicios del Estado en materia de servicios y asistencia social. Los Reales Decretos 3340/1983, de 23 de noviembre, y 1080/1984, de 29 de febrero, transfirieron competencias en materia de protección de la mujer y protección de menores, respectivamente. 
cia expresa no solo a las competencias ejecutivas en materia de Seguridad Social, sino también al papel que en materia de protección social juega la Seguridad Social. Esta opción contrasta con la que ha seguido alguna otra ley autonómica de «segunda generación» ${ }^{11}$. Por ejemplo, la Ley 14/2015, de 9 de abril, de Servicios Sociales de Extremadura, configura el derecho a los servicios sociales como «uno de los sistemas de protección social de los ciudadanos ante situaciones de necesidad que garantizan el bienestar social junto al sistema de Seguridad Social, el sistema de salud, el sistema educativo y las políticas de vivienda» ${ }^{12}$.

No obstante, la Ley 9/2016 define el sistema de servicios sociales como «el conjunto de servicios, recursos y prestaciones de las Administraciones Públicas de Andalucía, o vinculadas a las mismas, orientados a satisfacer el derecho a la protección social, en los términos recogidos en el Estatuto de Autonomía para Andalucía, en esta ley y en toda la normativa vigente en la materia» No debería resultar difícil integrar en este concepto los servicios y prestaciones de la Seguridad Social desde hace años transferidos a la Comunidad Autónoma.

\section{PRINCIPALES NOVEDADES QUE INTRODUCE LA LEY 9/2016, DE 27 DE DICIEMBRE}

Entre las principales novedades que aporta la Ley 9/2016 a la ordenación existente del Sistema Público de Servicios Sociales de Andalucía destaca la universalización de los derechos de todas las personas a las prestaciones y servicios de los servicios sociales del sistema, en condiciones de igualdad, con el fin de alcanzar su pleno desarrollo individual y social. Por tanto, el derecho a acceder a los servicios sociales se reconoce a todas las personas (arts. 1 y 9), con independencia de sus ingresos económicos, y

${ }^{11}$ En la misma línea que la ley andaluza, tampoco hay una sola referencia a la Seguridad Social en las Leyes 4/2009, de 11 de junio, de Servicios Sociales de las Illes Balears-Baleares; 5/2009, de 30 de junio, de Servicios Sociales de Aragón, y 7/2009, de 22 de diciembre, de Servicios Sociales de La Rioja; tampoco en la Ley 14/2010, de 16 de diciembre, de Servicios Sociales de Castilla-La Mancha, o en la Ley 16/2010, de 20 de diciembre, de Servicios Sociales de Castilla y León.

${ }_{12}$ En términos similares, la Ley 1/2003, de 24 de febrero, de Servicios Sociales del Principado de Asturias, no duda en recordar que el Sistema Público de Servicios Sociales regulado por la presente Ley tendrá carácter complementario en relación con las prestaciones de la Administración General del Estado en el ámbito de la Seguridad Social. 
no sólo a los que acrediten falta de medios económicos para subvenir a sus necesidades.

Ahora bien, la efectividad de esta universalidad de los servicios sociales ha de ponerse en relación con la financiación y con el catálogo de prestaciones (para cuya aprobación el Consejo de Gobierno tiene un plazo máximo de doce meses desde la entrada en vigor de la presente Ley, disposición adicional cuarta), pues en este catálogo deberán definirse cada uno de los servicios y prestaciones ofrecidas, su ámbito y alcance, las condiciones requeridas para acceder a los mismos, y su disponibilidad dentro del sistema.

La universalidad que incorpora la Ley 9/2016 es una universalidad subjetiva, que no objetiva (en atención a la protección que se dispensa). Sin ir más lejos, el art. 6, cuando regula los titulares del derecho a los servicios y prestaciones de este sistema público, incluye a «todas aquellas personas, no contempladas con anterioridad, que se encuentren en la Comunidad Autónoma en una situación de urgencia personal, familiar o social» ${ }^{13}$, para limitar la protección a que pueden tener acceso a las prestaciones del sistema «que permitan atender tales contingencias en los términos que se establezcan reglamentariamente» ${ }^{14}$.

La universalidad del derecho a acceder a los servicios sociales no equivale a gratuidad. La Ley 9/2016 no duda en establecer la participación de las personas usuarias de las prestaciones en su financiación en función de la naturaleza y coste de referencia del servicio, intensidad de utilización del mismo y de acuerdo con su capacidad económica ${ }^{15}$, por más que tam-

13 En referencia a los distintos supuestos de titularidad del derecho que regula el art. 6 de la Ley $9 / 2016$ son los siguientes:

«a) Todas las personas que tengan vecindad administrativa en Andalucía.

b) Las personas andaluzas en el exterior que podrán acceder a lo dispuesto en esta Ley en los términos previstos en la Ley 8/2006, de 24 de octubre, del Estatuto de los Andaluces en el Mundo.

c) Las personas menores de edad extranjeras que se encuentren en Andalucía.

d) Las personas con derecho de asilo a quienes se reconozca la condición de refugiado, o la protección subsidiaria, en los términos que establecen los tratados internacionales y la legislación en materia de extranjería».

${ }_{14}$ Aunque, en todo caso, tendrán garantizado el derecho a los servicios de información, valoración, diagnóstico y orientación, tanto a nivel primario como especializado.

${ }^{15}$ Según el art. 125.5 de la Ley 9/2016: «En el Catálogo de Prestaciones del Sistema Público de Servicios Sociales se determinarán las prestaciones que estarán exentas de aportación por parte de las personas usuarias, entre las que se encontrarán, en todo caso, las prestaciones de servicios de información, valoración, orientación, diagnóstico y asesoramiento, tanto en el nivel primario como en el especializado; la elaboración del Proyecto de Intervención Social; protección de menores de edad en situación de riesgo o desamparo; protección jurídica y social de las personas con capacidad limitada y de menores en situación de desamparo; prestaciones económicas específicas y directas orientadas a la erradica- 
bién se advierta que en ningún caso se pueda excluir a una persona de la prestación de servicios sociales financiados total o parcialmente con fondos públicos por el hecho de no disponer de recursos económicos o resultar éstos insuficientes.

Otro de los principales avances que introduce la Ley 9/2016 tiene que ver con la configuración del derecho a las prestaciones esenciales del sistema como un «derecho subjetivo de ciudadanía» exigible ante las Administraciones Públicas que ostentan las competencias en la gestión y provisión de las mismas ${ }^{16}$; en términos muy similares a los utilizados por las Leyes de Servicios Sociales de otras Comunidades Autónomas ${ }^{17}$. Ahora bien, la Ley «matiza» ese avance introduciendo una importante distinción entre las prestaciones garantizadas, que son las que se configuran como verdaderos derechos subjetivos, y las prestaciones condicionadas, que siguen sujetas al régimen de disponibilidad de recursos y a los criterios de prioridad en la asignación que objetivamente se establezcan (art. 43). Las personas titulares de las prestaciones garantizadas podrán reclamar su cobertura en vía administrativa y judicial de acuerdo con lo dispuesto en esta Ley, sus normas de desarrollo y en el resto de la legislación vigente aplicable a los servicios sociales (art. 7.3).

Las prestaciones garantizadas deberán describirse en el Catálogo de Prestaciones del Sistema Público de Servicios Sociales, con lo que su efec-

ción de la marginación y la desigualdad, y a la lucha contra la exclusión social; la protección y amparo a las personas víctimas de violencia de género o trata, así como, en su caso, su unidad de convivencia; el reconocimiento de la situación de discapacidad, determinando su tipo y grado; el tratamiento integral para las personas con problemas de drogodependencia y otras adicciones, así como cualquier otra que reglamentariamente se determine».

${ }_{16}$ Téngase en cuenta que la Ley 2/1998, de 4 de abril, se había limitado a incluir entre sus principios inspiradores el de responsabilidad pública: «mediante la adscripción por los poderes públicos de los recursos financieros, humanos y técnicos adecuados a la consecución de los objetivos propuestos» (art. 2.1). Es cierto que, según su exposición de motivos, este principio «genera un derecho subjetivo en favor del administrado», pero ni el «contenido de los servicios sociales» (art. 4) ni, en particular, el de las prestaciones económicas (art. 14) permitían configurarlos como verdaderos derechos subjetivos de los ciudadanos.

17 Por ejemplo, la Ley 12/2008, de 5 de diciembre, de Servicios Sociales de Euskadi, y también la Ley 14/2010, de 16 de diciembre, de Servicios Sociales de Castilla-La Mancha. Más recientemente la Ley 14/2015, de 9 de abril, de Servicios Sociales de Extremadura. Por su parte, la Ley 1/2003, de 24 de febrero, de Servicios Sociales del Principado de Asturias, incluye en su catálogo de prestaciones aquellas «que tendrán el carácter de fundamentales y que serán exigibles como derecho subjetivo en los términos establecidos en el mismo directamente o previa indicación técnica y prueba objetiva de su necesidad, con independencia, en todo caso, de la situación económica de los beneficiarios». Como un cambio de tendencia respecto de lo que había venido sucediendo lo interpretaron J. ToRNOS Mas y A. GaLÁn GALÁn, «La configuración de los servicios sociales como servicio público...», op. cit., p. 29. 
tividad jurídica, salvo excepciones que remiten a la normativa específica ${ }^{18}$, queda sujeta a la aprobación y publicación de ese Catálogo (art. 42.2 y disposición transitoria segunda), al que la Ley, no obstante, atribuye un contenido mínimo, esto es, una relación de prestaciones que deberán incluirse en todo caso (art. 42) ${ }^{19}$. De esta forma puede decirse que el margen de discrecionalidad de la Administración en la concesión de las prestaciones se reduce al menos respecto del que tenía con la Ley 2/1988.

Una vez reconocido ese derecho subjetivo a los servicios sociales (art. 7), la Ley incorpora, en su título I, una referencia a los derechos y obligaciones que dotan de contenido al concepto de ciudadanía cuando se proyecta en este ámbito. En concreto, en el primer capítulo de este título

${ }_{18}$ En concreto, las prestaciones contempladas en la Ley 39/2006, de 14 de diciembre, y en su normativa de desarrollo, y el reconocimiento de la situación de discapacidad, determinando su tipo y grado.

${ }^{19}$ En efecto, según el art. 42.2: «El Catálogo de Prestaciones del Sistema Público de Servicios Sociales describirá de forma clara las prestaciones garantizadas, entre las que, al menos, estarán:

a) Los servicios de información, valoración, orientación y asesoramiento.

b) La elaboración y ejecución del Proyecto de Intervención Social, a fin de garantizar una adecuada atención acorde con la valoración social de la persona, familia o unidad de convivencia, donde se incorporarán los objetivos a alcanzar, los medios disponibles, los plazos máximos de tramitación y ejecución, así como las acciones específicas orientadas a fomentar, en su caso, la inclusión personal, social, educativa y laboral.

c) El servicio de teleasistencia.

d) La atención inmediata en situaciones de urgencia y emergencia social.

e) Los servicios específicos para la protección de niños o niñas en situación de riesgo o desamparo.

f) La protección jurídica y social de las personas con capacidad limitada y de personas menores de edad en situación de desamparo.

g) Las prestaciones económicas específicas y directas orientadas a la erradicación de la marginación y la desigualdad y a la lucha contra la exclusión social, que deberán incorporar un itinerario a través de un plan de inclusión y/o inserción sociolaboral.

b) Las prestaciones contempladas en la Ley 39/2006, de 14 de diciembre, y en su normativa de desarrollo.

i) La protección y amparo a las personas víctimas de violencia de género o trata, así como, en su caso, a su unidad de convivencia.

j) El reconocimiento de la situación de discapacidad, determinando su tipo y grado.

k) El servicio de ayuda a domicilio de los servicios sociales comunitarios no vinculados a la Ley 39/2006, de 14 de diciembre, de Promoción de la Autonomía Personal y Atención a las Personas en Situación de Dependencia.

l) El alojamiento alternativo.

m) La prestación de servicios de apoyo psicosocial y psicoeducativo de atención a la infancia y la familia.

n) El tratamiento integral para las personas con problemas de drogodependencia y otras adicciones.

n) Atención a personas mayores víctimas de violencia intrafamiliar». 
se distingue entre los derechos vinculados al acceso a los servicios sociales, que identifica como «derechos de la ciudadanía en relación con los servicios sociales» (art. 9), y los derechos del usuario, que le corresponden una vez admitido en el servicio. Los primeros incluyen, además de las facultades de admisión y acceso a prestaciones, las de participar en la definición, evaluación, planificación, etc., de los servicios; las de acceder a los cauces de información, sugerencias y quejas que permitan el ejercicio efectivo de los derechos y su defensa, y las de dar instrucciones previas, para situaciones futuras de limitación de la capacidad de obrar, respecto a la asistencia y cuidados que se les puedan procurar (art. 9). Los segundos, en cambio, tienen que ver con las prestaciones y servicios, asistencia e información a la que se tiene derecho una vez que, como se ha dicho, se accede a los servicios (art. 10). Esta distinción es importante, pues se entiende que la posición del ciudadano se encuentra mucho más reforzada cuando ya ha sido efectivamente admitido $^{20}$. Por último, junto a estos derechos, la Ley regula, en particular, los derechos de las personas residentes en centros de servicios sociales de la Comunidad Autónoma.

En el título II de la Ley se abordan todos los aspectos de ordenación y organización necesarios para configurar y desarrollar este Sistema Público de Servicios Sociales de Andalucía. En concreto, el sistema se sigue organizando conforme a dos criterios: funcional y territorial. Por lo que se refiere al primero de esos criterios, baste señalar que se sigue manteniendo un nivel primario con los «servicios sociales comunitarios» como estructura básica, y un nivel especializado en el que se ubican los «servicios sociales especializados». La organización y gestión de los servicios sociales comunitarios y de sus centros se atribuye a las entidades locales en el ejercicio de las competencias propias en materia de servicios sociales que les confiere el Estatuto de Autonomía y la Ley 5/2010, de 11 de junio, de Autonomía Local. La Ley 9/2016 ofrece una regulación mucho más detallada sobre sus funciones y organización, en la que destaca la incorporación de la figura del profesional de referencia que se asignará a cada persona y, en su caso, unidad de convivencia que acceda al Sistema Público de Servicios Sociales, «al objeto de garantizar el carácter integral de los itinerarios de atención y su continuidad» (art. 31). Los servicios sociales especializados, por su parte, dejan de estar definidos por sus destinatarios ${ }^{21}$ y pasan a

\footnotetext{
${ }^{20}$ Sobre el tema vid. V. Aguado i Cudolà, «El régimen jurídico...», op. cit., p. 66.

${ }^{21}$ En efecto, el art. 11 de la Ley 2/1988 definía a los servicios sociales especializados como aquellos que se dirigen hacia determinados sectores de la población que por sus circunstancias necesitan de una atención específica.
} 
estarlo por la mayor complejidad de las actuaciones que han de desarrollar y la especialización técnica que requieren (art. 32).

La estructura territorial del sistema continúa respondiendo al principio de descentralización (progresivo desplazamiento de la gestión de los servicios sociales hacia los órganos e instituciones más próximos al usuario), al que se suman algunos de los principios que según el Estatuto de Autonomía (art. 90 de la Ley Orgánica 2/2007) rigen la organización territorial de Andalucía (desconcentración, coordinación) (art. 36). Con todo, entre esos principios orientadores de la organización territorial del sistema de servicios sociales que formula el art. 36 de la Ley aparecen formulados como tales los que no dejan de ser objetivos de esa organización territorial: la eficacia y eficiencia en la satisfacción de las necesidades sociales, coordinación y trabajo en red, proximidad a la ciudadanía.

La Ley dedica un capítulo de este título II a regular las competencias de las Administraciones Públicas. En efecto, según anuncia la exposición de motivos de la Ley, en la planificación del Sistema Público de Servicios Sociales de Andalucía están llamados a jugar un papel muy importante el conjunto de las Administraciones Públicas en colaboración y coordinación con el resto de agentes sociales que se integran de pleno derecho en el sistema y complementan el marco de actuación de los poderes públicos. En concreto, respecto de las entidades locales destaca la regulación de unas competencias propias (art. 51), junto a las que pueden asumir por delegación, respecto de los servicios sociales de titularidad autonómica, en virtud de los principios de descentralización, subsidiariedad, proximidad y eficiencia, en el marco de la Ley 5/2010, de 11 de junio.

Con el fin de garantizar una prestación ágil y eficaz de los servicios sociales, las Administraciones Públicas actuantes en el ámbito territorial de la Comunidad Autónoma se prestarán entre sí la colaboración necesaria mediante los instrumentos de cooperación previstos en la Ley 40/2015, de 1 de octubre, de Régimen Jurídico del Sector Público; en la Ley 9/2007, de 22 de octubre, de la Administración de la Junta de Andalucía, en la Legislación Estatal y Autonómica de Régimen Local, así como en las leyes autonómicas que han incidido sobre parcelas concretas de la política social (como la Ley 13/2007, de 26 de noviembre, de Medidas de Prevención y Protección Integral contra la Violencia de Género) (art. 55).

La exposición de motivos ha querido resaltar también, como mérito de esta Ley, el papel que asigna a la «estrategia global de calidad, eficiencia y sostenibilidad», que se elaborará con la participación de la Administración y de las organizaciones empresariales y sindicales más representativas. 
Esta estrategia deberá incorporar los objetivos a alcanzar «en los ámbitos de ciudadanía, profesionales y organización», y desarrollará «indicadores» de seguimiento y evaluación de la calidad. Se trata de una actuación que se suma a otras que también se contemplan en la Ley ${ }^{22}$, con las que se pretende avanzar en la «planificación, calidad, eficiencia y sostenibilidad del sistema» (título III). Esta actuación implica a todas las personas titulares de los recursos y servicios, e involucra de forma muy especial al conjunto de profesionales que desarrollan sus funciones en el sistema (a los que se refieren los arts. 59 a 63 ).

Otra de las novedades que introduce la Ley tiene que ver con la ordenación del papel de la iniciativa privada en materia de servicios sociales ${ }^{23}$. En este punto, la Ley 2/1988 sólo contemplaba la colaboración de la iniciativa social a través de las entidades privadas sin ánimo de lucro (art. 25), que sujetaba al cumplimiento de unos requisitos, y regulaba el apoyo al voluntariado social que colabora con las Administraciones Públicas y con la iniciativa social en las tareas de prestación de servicios sociales (art. 26). Años después, la Ley 15/2001, de 26 de diciembre, de Medidas Fiscales, Presupuestarias, de Control y Administrativas, modificó el art. 25 de la Ley 2/1988 permitiendo la colaboración, sin restricción alguna, de las entidades privadas con ánimo de lucro en el ámbito del Sistema Público de Servicios Sociales, siempre que cumplieran los mismos requisitos que se venían exigiendo a las entidades privadas sin ánimo de lucro.

Lo que ahora hace la Ley 9/2016, supuestamente para fortalecer y mejorar el Sistema Público de Servicios Sociales (exposición de motivos), es desarrollar el papel que esa iniciativa privada puede jugar estableciendo un marco normativo general para su actividad y las condiciones de su participación (art. 1). En concreto, la Ley dedica todo un título (IV) a regular esta iniciativa privada y social en la provisión de servicios sociales en

\footnotetext{
${ }^{22}$ Como la planificación de las estrategias, recursos, servicios y prestaciones de servicios sociales que ha de llevar a cabo la Consejería (art. 73); la mejora de la calidad (art. 78); la coordinación con las funciones que corresponden a otros sistemas de protección social afines o complementarios (art. 54); la creación de la «Red Andaluza de Investigación de Políticas Sociales» (art. 88) para la investigación e innovación en materia de políticas sociales, o la «inspección de servicios sociales».

${ }_{23}$ Téngase en cuenta que, hasta ahora, la iniciativa privada respecto de los servicios sociales en Andalucía era posible con apoyo en la iniciativa social y empresarial, y, más en particular, en la iniciativa mercantil y la libertad de empresa, que parecen excluir la reserva al sector público en régimen de monopolio de los servicios sociales. En estos términos vid. F. J. VILlaR ROJAS, «Iniciativa privada y prestación de servicios sociales. Las redes o sistemas públicos de servicios sociales», en A. EzQuerRA Huerva (coord.), El marco jurídico de los servicios sociales en España, Barcelona, Atelier, 2012, p. 91.
} 
Andalucía, sujetándola al régimen de autorización, acreditación y registro, y reconociendo su papel en la configuración del sistema público mediante relaciones de colaboración admitidas en ley (exposición de motivos) ${ }^{24}$. Algunas de esas disposiciones extienden su aplicación a los servicios sociales de iniciativa privada no incluidos en el sistema (art. 4) ${ }^{25}$. Estas entidades que operan fuera del Sistema Público de Servicios Sociales, además de quedar, de esta forma, sujetas a regulación y control, podrán ser destinatarias de subvenciones y otras ayudas públicas conforme a la normativa vigente (art. 112) ${ }^{26}$.

Esta regulación de la iniciativa privada se completa con varios capítulos que se dedican a ordenar los instrumentos y fórmulas a través de los que articular su participación en la provisión de servicios del Sistema Público de Servicios Sociales de Andalucía ${ }^{27}$.

${ }^{24}$ El régimen jurídico de los servicios sociales que establece esta Ley y, en concreto, el régimen de autorización y acreditación administrativa y registro de entidades, centros y servicios se enmarca en las exigencias de la Directiva 2014/24/UE sobre contratación pública, que, según advierte la exposición de motivos de la Ley 9/2016, ha permitido abrir nuevas posibilidades respecto de la organización de los servicios a las personas. En concreto, la Directiva 2014/24/UE da libertad a los Estados miembros y a los poderes públicos para prestar por sí mismos esos servicios u organizar los servicios sociales de manera que no sea necesario celebrar contratos públicos, por ejemplo, mediante la simple financiación de estos servicios o la concesión de licencias o autorizaciones a todos los operadores económicos que cumplan las condiciones previamente fijadas por el poder adjudicador, sin límites ni cuotas, siempre que dicho sistema garantice una publicidad suficiente y se ajuste a los principios de transparencia y no discriminación (considerando 114).

${ }_{25}$ Al igual que ya se había hecho, por ejemplo, en la Ley 12/2007, de 11 de octubre, de Servicios Sociales en Cataluña. En concreto, a estos servicios sociales de iniciativa privada le serán de aplicación las disposiciones de la Ley 9/2016 que regulan los derechos y obligaciones de las personas usuarias y profesionales que se definan, así como la autorización, el registro y la inspección y control de entidades, servicios y centros, como garantía del cumplimiento de los requisitos materiales, funcionales y de personal que les sean de aplicación (art. 4.2).

${ }^{26}$ Ahora bien, esta posibilidad queda circunscrita a las entidades de iniciativa social para contribuir a la realización de actividades de servicios sociales, siempre que tales actividades se correspondan con los fines propios de la entidad, quede debidamente justificado el interés social de la actuación a subvencionar y no supongan un menoscabo de la prestación pública del servicio.

${ }^{27}$ En concreto el concierto social, al que se define a los efectos de esta Ley como «el instrumento por medio del que se produce la prestación de servicios sociales de responsabilidad pública a través de entidades cuya financiación, acceso y control sean públicos» (art. 101). Asimismo, la Ley prevé las modalidades de partenariado (fórmula de colaboración entre entidades empresariales y el Sistema Público de Servicios Sociales, art. 113.2), patrocinio (con el objeto de establecer una relación táctica con empresas que quieran participar de forma puntual en acciones concretas organizadas por el Sistema Público de Servicios Sociales) y mecenazgo (participación de personas físicas que quieran colaborar al sostenimiento del sistema público mediante la aportación de fondos o la entrega o puesta a disposición de bienes, art. 113.2) en los servicios sociales de Andalucía, determinando sus condiciones básicas. 
En el título V, relativo a la «Financiación de los servicios sociales», la novedad se concreta en que se introduce en el Sistema Público de Servicios Sociales de Andalucía la gestión sobre la base de contratos-programa como «una de las herramientas de la que se dota el Sistema Público de Servicios Sociales de Andalucía para vincular la financiación del sistema al cumplimiento de los fines y objetivos del mismo sobre la base de la calidad y la eficiencia del sistema» (art. 3). El objetivo es mejorar la gestión, y más en particular mejorar la eficiencia, la transparencia y el rendimiento de cuentas, en un Sistema Público de Servicio Sociales que ha pasado a concebir la prestación de esos servicios como un derecho de los ciudadanos. En concreto, el art. 117 de la Ley 9/2016 prevé el establecimiento por la Consejería competente en materia de servicios sociales de un contrato-programa con las entidades instrumentales involucradas en la provisión de servicios sociales en el que se fijarán las directrices de actuación, los objetivos a alcanzar y los recursos que para ello se asignan. En los contratos-programa se establecerán, a su vez, los indicadores necesarios que posibiliten el seguimiento del grado de realización de los objetivos definidos. Estos contratos se ven como una forma adecuada de canalizar los recursos y asegurar que su finalidad se orienta al cumplimiento de los objetivos que en cada momento se consideren esenciales para la sostenibilidad y la cohesión del mismo.

Por último, la Ley 9/2016 ofrece una regulación del régimen de infracciones y sanciones (título VI) más completa que la que se contenía en la Ley 2/1988 que, como el resto de leyes autonómicas de «primera generación» en materia de servicios sociales, era muy exigua en el tratamiento de la cuestión. Esta Ley no establecía las autoridades encargadas de instruir el necesario expediente y de acordar la sanción oportuna, lo que, en la práctica, impidió la adopción de medidas sancionadoras ${ }^{28}$.

La regulación que ahora se ofrece pretende «mantener los estándares adecuados para el funcionamiento del sistema dentro de las mayores garantías y de la necesaria seguridad jurídica de las partes». A tal efecto el título VI de la Ley incorpora, en un primer capítulo, una exhaustiva tipificación de faltas, una precisión de la responsabilidad en juego y unas reglas sobre prescripción; establece en el segundo capítulo las sanciones, los criterios para su graduación y otras reglas para su aplicación, incluida la refe-

${ }^{28}$ Así lo describe S. FERnÁNDEZ RAmos, «La disciplina en materia de servicios sociales», en A. EzQuerra Huerva (coord.), El marco jurídico de los servicios sociales en España, Barcelona, Atelier, 2012, p. 178. 
rencia a los órganos competentes para iniciar el procedimiento sancionador, cuya regulación se desarrolla en el tercer capítulo.

Este régimen sancionador se suma a los mecanismos de control (autorización, acreditación administrativa y registro de entidades, centros y servicios sociales) y a la función inspectora en materia de servicios sociales, que la Ley ha puesto en relación con la «planificación, calidad, eficiencia y sostenibilidad del sistema» (título III). A la Consejería competente en materia de servicios sociales le corresponde «ejercer la potestad inspectora para garantizar el adecuado cumplimiento de las disposiciones legales y reglamentarias ordenadoras de los servicios sociales (art. 50).

\section{LA RELACIÓN DE LA LEY 9/2016 CON OTRAS LEYES DE ÁMBITO ESTATAL Y AUTONÓMICO}

La Ley 9/2016 se apoya, como ya se ha dicho, en la competencia exclusiva que el Estatuto de Autonomía atribuye a la Comunidad Autónoma en materia de servicios sociales, pero, como en algún momento de su exposición de motivos reconoce, en determinadas áreas de actuación la Ley se mueve también, directamente en unos casos, indirectamente en otros ${ }^{29}$, en el marco regulado a nivel estatal por normas dictadas al amparo del art. 149.1.1 CE, que atribuye al Estado competencia exclusiva para regular «las condiciones básicas que garanticen la igualdad de todos los españoles

29 Por ejemplo, la Ley 9/2016 menciona expresamente, como desarrollo de la Ley 2/1988, la Ley 13/2007, de 26 de noviembre, de Medidas de Prevención y Protección Integral contra la Violencia de Género. Esta Ley recoge los principios y fines que han de guiar la actuación de los poderes públicos de Andalucía tendente a la erradicación de la violencia de género, entre los que destacan «reforzar hasta la consecución de los mínimos exigidos por los objetivos de la Ley los servicios sociales de información, de atención, de emergencia, de apoyo y de recuperación integral, así como establecer un sistema para la más eficaz coordinación de los servicios ya existentes a nivel municipal y autonómico», «garantizar el acceso a las ayudas económicas que se prevean para las mujeres víctimas de violencia de género y personas de ellas dependientes» o «establecer un sistema integral de tutela institucional en el que la Administración andaluza, en colaboración con la Delegación Especial del Gobierno contra la Violencia sobre la Mujer, impulse la creación de políticas públicas dirigidas a ofrecer tutela a las víctimas de la violencia contemplada en la presente Ley». Pues bien, estos fines y principios de la Ley 13/2007, por lo que aquí interesa, no difieren de los fines y principios que incorporó la Ley Orgánica 1/2004, entre ellos, «fortalecer las medidas de sensibilización ciudadana de prevención, dotando a los poderes públicos de instrumentos eficaces en el ámbito educativo, servicios sociales, sanitario, publicitario y mediático». De hecho, la Ley 13/2007 no dudó en invocar la Ley Orgánica 1/2004, a la que calificó como «el instrumento que cumple decididamente con las recomendaciones y directrices internacionales y de ámbito regional europeo». 
en el ejercicio de los derechos y en el cumplimiento de los deberes constitucionales», y, de esa forma, intervenir en materia de asistencia social.

En definitiva, no parece que esa atribución a la Comunidad Autónoma de competencias exclusivas en materia de asistencia social pueda impedir la intervención del Estado, que ha seguido asumiendo competencias, con apoyo en el art. 149.1.1 CE, en alguna parcela concreta. En efecto, como se recordará, la Ley 39/2006, de 14 de diciembre, de Promoción de la Autonomía Personal y Atención a las Personas en Situación de Dependencia, se aprobó con apoyo en la competencia exclusiva del Estado para la regulación de las condiciones básicas que garanticen la igualdad de todos los españoles en el ejercicio de los derechos y en el cumplimiento de los deberes constitucionales (art. 149.1 CE). Una intervención del legislador criticada por algún sector doctrinal ${ }^{30}$, pero aceptada, en general, por las leyes autonómicas en materia de servicios sociales.

Así, la Ley 9/2016 remite a la Ley 39/2006, de 14 de diciembre, a propósito de quiénes son titulares del derecho a acceder a las prestaciones y servicios sociales enmarcados en el sistema para la autonomía y atención a la dependencia (art. 6.3), e incluye entre las prestaciones garantizadas, que ha de describir el catálogo de prestaciones, las contempladas en la Ley 39/2006 y en su normativa de desarrollo [art. 42.2.h)]. Además, la disposición adicional segunda de la Ley 9/2016 modifica la Ley 6/1999, de 7 de julio, de Atención y Protección a las Personas Mayores de Andalucía, precisamente para adecuar la denominación y clasificación de los recursos para la atención a las personas mayores a las que establece la Ley 39/2006, de 14 de diciembre.

También se ha ocupado de incorporar el enfoque de género en todo el modelo de intervención de los servicios sociales, evitando la feminización de la acción social en toda su extensión; enfoque que había sido asumido como principio general de actuación de los poderes públicos en Andalucía por la Ley 12/2007, de 26 de noviembre, para la Promoción de la Igualdad de Género en Andalucía ${ }^{31}$. Aunque no lo haya querido recordar la

\footnotetext{
${ }^{30}$ V. Aguado i Cudolà, «El régimen jurídico...», op. cit., p. 51.

${ }^{31}$ En concreto, la Ley propone transcender de la categoría mujer como proveedora de cuidados en su rol tradicional de cuidadora, «reconociendo el valor humano de la provisión de cuidados, pero a su vez reivindicando el reparto igualitario de esta tarea entre mujeres y hombres». Para ello consagra como principio rector el principio de «transversalidad de género», según el cual «los poderes públicos potenciarán que la perspectiva de la igualdad de género esté presente en la elaboración, ejecución y seguimiento de las disposiciones normativas, de las políticas en todos los ámbitos de actuación, considerando sistemáticamente las prioridades y necesidades propias de las mujeres y de los hombres, teniendo en
} 
Ley 9/2016, esta Ley 12/2007 se dictó siguiendo el marco de desarrollo del principio de igualdad de trato de la Ley Orgánica 3/2007, de 22 de marzo, para la Igualdad Efectiva de Mujeres y Hombres.

Tampoco hay en la Ley 9/2016 ninguna referencia expresa al Real Decreto Legislativo 1/2013, de 29 de noviembre, por el que se aprueba el Texto Refundido de la Ley General de Derechos de las Personas con Discapacidad y de su Inclusión Social, según el cual «las Administraciones Públicas velarán por el mantenimiento de unos servicios de atención adecuados mediante la coordinación de los recursos y servicios de habilitación y rehabilitación», entre otros, en el ámbito de los servicios sociales. Pese a esa omisión de la Ley 9/2016, la Comunidad Autónoma debe tener en cuenta, de un lado, que del art. 11 de este texto normativo se desprende con claridad que «la prevención de deficiencias y de intensificación de discapacidades constituye un derecho y un deber de todo ciudadano y de la sociedad en su conjunto», y por ello ha de formar parte «de las obligaciones prioritarias del Estado en el campo de la salud pública y de los servicios sociales», y, de otro, que esta norma no ha dudado en asignar un papel complementario, de las medidas específicas que regula ${ }^{32}$, a los servicios y prestaciones económicas para las personas con discapacidad y sus familias que se puedan regular en las Comunidades Autónomas (art. 50).

cuenta su incidencia en la situación específica de unas y otros, al objeto de adaptarlas para eliminar los efectos discriminatorios y fomentar la igualdad de género». Más aún, una de las funciones de los servicios sociales comunitarios, según el art. 28, «será el estudio de la realidad social y análisis de necesidades y demanda de servicios sociales. En la evaluación de resultados se tendrá en cuenta el diferente impacto entre mujeres y hombres, y se aplicará la perspectiva de género para facilitar la no perpetuación de roles de género. Asimismo, en el seguimiento, determinación de prestaciones y recursos, y en la evaluación de resultados se tendrá en cuenta el diferente impacto entre mujeres y hombres, y se aplicará la perspectiva de género para facilitar la no perpetuación de roles de género».

32 En concreto, el art. 50 del Real Decreto Legislativo 1/2013 reconoce a «las personas con discapacidad y sus familias» el derecho a los servicios sociales de apoyo familiar, de prevención de deficiencias y de intensificación de discapacidades, de promoción de la autonomía personal, de información y orientación, de atención domiciliaria, de residencias, de apoyo en su entorno, servicios residenciales, de actividades culturales, deportivas, ocupación del ocio y del tiempo libre». 


\section{REFLEXIONES FINALES}

No cabe duda que la Ley 9/2016 es una norma que se mueve más bien en la línea de definir un marco de actuación, de establecer principios y reglas generales, y pautas de organización y funcionamiento del sistema, que de precisar o concretar recursos, actuaciones y prestaciones. A esta conclusión lleva la amplitud a la que recurre la Ley a la hora de identificar las necesidades sociales que el Sistema Público de Servicios Sociales ha de proteger y cubrir adecuadamente (art. 2): «Las carencias de las personas en el acceso a unos recursos que garanticen unas condiciones de vida dignas; contar con un adecuado entorno de convivencia personal, familiar y social; poder integrarse plenamente en la sociedad; alcanzar la plena autonomía personal, tanto en lo relativo a las carencias materiales como a las funcionales, y disponer de cauces para la participación social». Sin que contribuya demasiado a su precisión que la Ley añada que se trata de necesidades sociales que derivan «de la interacción de las personas con su entorno, sea este grupal o comunitario».

Esta amplitud se repite en la formulación de algunos de los objetivos de la política de servicios sociales de Andalucía (art. 5), entre otros, «detectar situaciones de necesidades sociales y personales, elaborar las estrategias para su cobertura y adoptar las medidas adecuadas para favorecer el bienestar social y mejorar la calidad de vida de las personas, las familias y los grupos sociales, con especial atención a las situaciones de desprotección y desamparo», o «promover la igualdad efectiva de las personas, eliminando discriminaciones o estigmas por razón de sexo, orientación sexual, identidad de género, origen de las personas, discapacidad o por cualquier otra condición o circunstancia personal o social».

Lo mismo podría decirse de las referencias que se contienen en su articulado a «otros sistemas de protección social», por ejemplo, cuando se establecen las funciones de los servicios sociales comunitarios, entre las que destacan la información, valoración, orientación y asesoramiento a la población sobre los posibles recursos de otros sistemas de protección social, y, en particular, la coordinación con los servicios sanitarios, educativos, de empleo, de vivienda y de justicia; así como la coordinación y el trabajo de red con los equipos profesionales de los demás sistemas de protección social (art. 28). En concreto, a «las prestaciones previstas en otros sistemas de protección social» hace referencia el art. 46.1 de la Ley para establecer que se incluyan en el Proyecto de Intervención Social, que es la 
actuación diseñada para garantizar el carácter integral de la atención y su continuidad, de acuerdo con la valoración diagnóstica de las necesidades que se haya hecho. Según el art. 50 de la Ley 9/2016 corresponde a la Consejería competente en materia de servicios sociales elaborar y desarrollar protocolos de coordinación con otros sistemas de protección social que puedan confluir con el Sistema Público de Servicios Sociales de Andalucía en áreas concretas de la intervención social, a la que corresponde también suscribir convenios de colaboración en materia de servicios sociales con la Administración del Estado o con la Administración local.

En las ocasiones en las que la Ley entra a precisar de qué otros sistemas de protección social está hablando menciona siempre al sistema de salud, educativo, judicial, de empleo o de vivienda. Así sucede en el art. 58 de la Ley, que precisamente regula la coordinación entre los servicios sociales y otros sistemas de protección social, según el cual «los órganos de las Administraciones Públicas competentes en materia de servicios sociales deberán coordinar sus actuaciones con las de los órganos competentes para la prestación de los servicios que corresponden a otros sistemas y políticas públicas, en particular con el sistema de salud, con el sistema educativo, con el sistema judicial, con las políticas de empleo, inserción laboral y formación, vivienda, accesibilidad, e igualdad, y, en general, con cualesquiera otras políticas públicas que pudieran confluir con los servicios sociales en áreas concretas de la intervención social».

No hay, por tanto, ninguna referencia expresa al sistema de Seguridad Social, por más que pueda entenderse incluido en esa referencia general a cualesquiera otras políticas públicas. Sin embargo, es evidente que el Sistema Público de Servicios Sociales de Andalucía, como los que se han creado y regulado en el resto de Comunidades Autónomas, se ha de integrar en un sistema mucho más complejo de protección social en el que el sistema público de Seguridad Social juega un papel importante ${ }^{33}$.

Tal vez esa omisión que hace la Ley de la protección social que proporciona la Seguridad Social pueda disculparse porque estos sistemas autonómicos de servicios sociales tienen una función (de prevención, atención o cobertura de las necesidades individuales y sociales básicas de las personas en su entorno, con el fin de alcanzar o mejorar su bienestar) ${ }^{34}$ que

33 Como dijera la STC 239/2003, este régimen de protección social que el art. 41 CE encomienda a los poderes públicos constituye el eje fundamental del sistema de protección social.

${ }^{34}$ En otras leyes autonómicas se habla de «prevención y eliminación de los factores etiológicos que conducen a situaciones de marginación social» y «asegurar el derecho de las per- 
supera la propia del sistema de la Seguridad Social. Ahora bien, esta circunstancia no justifica que los poderes públicos implicados no hagan un esfuerzo más claro por articular y coordinar las distintas vías que integran ese macrosistema de protección social. No hacerlo puede terminar siendo una irresponsabilidad, máxime si se tiene en cuenta que la expansión de los servicios sociales, junto a la asistencia social, como manifestaciones de una acción social de los poderes públicos que extiende la protección a ámbitos y sujetos a los que no llega el sistema de Seguridad Social, puede servir de «excusa» para no seguir avanzando también en la ampliación y mejora de los niveles de suficiencia de la acción protectora que dispensa el sistema público de Seguridad Social y en el desarrollo de los principios que según el art. 2 TRLGSS lo ordenan (de universalidad, unidad, solidaridad e igualdad). Además, de confirmarse esa posibilidad habría que preguntarse si con esa ordenación de los servicios sociales, exclusivamente en el ámbito autonómico, queda realmente asegurada la igualdad de todos los españoles en el acceso a este nivel de protección social.

sonas a vivir dignamente durante todas las etapas de su vida, teniendo cubiertas las necesidades sociales». En estos términos, por ejemplo, Ley 1/2003, de 24 de febrero, de Servicios Sociales del Principado de Asturias; Ley 12/2008, de 5 de diciembre, de Servicios Sociales de Euskadi; Ley 14/2010, de 16 de diciembre, de Servicios Sociales de Castilla-La Mancha, y Ley 14/2015, de 9 de abril, de Servicios Sociales de Extremadura. 
